Ann. Zootech., 1981, 30 (4), 391-410

\author{
I.N.R.A. \\ Bibliotheque Uo : $?$ \\ Domaime de Crojell. \\ 63039 \\ Clermont-FD Cedex?
}

\title{
Préférences alimentaires et comportement de la larve de daurade Sparus auratus face à du zooplancton congelé
}

\author{
Maroudio KENTOURI, P. DIVANACH et J. PARIS \\ Station de Biologie Marine et Lagunaire \\ Quai de Bosc-Prolongé \\ F 34200 Sète
}

\section{Résumé}

Cinq essais d'élevage sont effectués dans des bassins circulaires de 570 1. Depuis le stade de l'ouverture de la bouche jusqu'à 92 jours, des larves de daurades sont nourries avec du zooplancton congelé. Les taux de survie et la croissance sont rapportés dans la figure 1. Lorsque l'alimentation initiale est constituée de Brachionus plicatilis de grande taille $(261 \pm 47 \mu)$ et/ou d'œufs de Rotifères, la mortalité est totale. Par contre, lorsque des Rotifères plus petits $(222 \pm 45 \mu)$ et/ou des cufs de Mytilus galloprovincialis sont distribués pendant les premiers jours, les résultats sont comparables à ceux rapportés par BarNaBE 1976 et Ramos 1978 qui utilisent des proies vivantes.

La première prise de nourriture est plus précoce avec des proies de petite taille (fig. 2) et jusqu'à $4,5 \mathrm{~mm}$, les larves sélectionnent préférentiellement les proies inférieures à $200 \mu$ (fig. $2 \mathrm{D}$ et fig. 3 ). Les Rotifères sont digérés en moins de 2 heures (fig. $5 \mathrm{~A} ;$ tab. 5) mais lorsque les larves ne disposent pas de petits Rotifères, elles transfèrent leur activité de chasse vers des oufs de Rotifères (tab. 3 et 4) qui ne sont pas digérés (fig. 5 B ; tab. 5) ou des bulles d'air de taille adéquate Les caractéristiques de ce comportement sont rapportées dans la figure 4.

L'ingestion de bulles n'est pas léthale car elles sont excrétées en moins de 2 heures (fig. $4 \mathrm{E}$ ), mais ce phénomène amène une purge du tractus digestif (fig. $4 \mathrm{~F}$ ).

Deux mécanismes de sélection des proies par les larves sont décrits (tab. 1 à 4 ) l'abandon après observation étant plus fréquent que la régurgitation.

Les mécanismes comportementaux décrits permettent d'expliquer une des causes de mortalité pendant l'élevage larvaire de Sparus auratus.

\section{1. - Introduction}

Grâce à la bonne qualité de sa chair, son adaptation facile à la captivité et sa croissance rapide (Koenig, 1974; Rene, 1974 a et b ; Porter, 1979), la daurade Sparus auratus se place parmi les poissons d'aquaculture marine les plus prometteurs. Malheureusement, alors que les techniques de production d'alevins de loup Dicentrarchus labrax ou de sole Solea solea semblent maîtrisés (GIRIN, 1979), l'élevage de 
la daurade est actuellement limité par des mortalités importantes pendant la phase larvaire (BARNABE, 1976; RAmOS, 1978) et la production de juvéniles n'est l'ouvre que de quelques-uns (Arias \& Pascual, 1979, Rapport C.G.P.M., 1980). Au Japon, la réussite de l'élevage de la daurade Pagrus major au stade quasi industriel (FABRE, 1979) est en partie liée à une connaissance approfondie du comportement alimentaire et des besoins de ses stades larvaires (Fukuhara, 1974; Kitajima, 1976 ; Kitajima et al., 1976; Fukusho, Hara, Iwamoto, 1977; Kitajima, 1979). De tels travaux n'existent pas pour Sparus auratus. La présente contribution rapporte les résultats obtenus au cours de cinq essais d'élevage de larves de daurade avec du plancton congelé et met en évidence l'influence des facteurs comportementaux sur la réussite de l'opération.

\section{2. - Matériel et méthode}

Les essais sont réalisés dans 5 bassins circulaires de 5701 en polyéthylène blanc disposés en une batterie rectiligne permettant une observation directe aisée. L'éclairage zénithal permanent est fourni par une double rampe de tubes fluorescents de $60 \mathrm{w}$, installés de façon à suprimer les reflets. Le circuit d'eau est du type semi-fermé. L'apport d'eau neuve passe progressivement de 1 p. 100 par jour pendant la phase pré-larvaire à 200 p. 100/jour à 90 jours. L'eau recyclée est épurée sur un filtre biologique représentant 32 p. 100 du volume d'élevage et dont le substrat $\left(480 \mathrm{~m}^{2}\right.$ d'interface solide-liquide) est constitué de copeaux de polyéthylène. Le taux de recyclage passe de 0,7 p. 100 en début d'élevage à 14 fois le volume par jour au bout de 40 jours.

Les œufs proviennent de géniteurs maintenus en captivité depuis plus de 4 ans. La ponte est provoquée par injection d'HCG à 800 U.I./kg. L'incubation a lieu dans

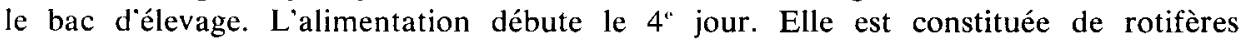
(Brachionus plicatilis et Brachionus sp.) et de copépodes (Eurytemora velox) pêchés dans les lagunes de la région avec les techniques décrites par BARNABE (1979) et Divanach, Barnabe \& Jullian (1980) ainsi que d'œufs de moules Mytilus galloprovincialis dont la ponte est induite par $\mathrm{H}_{2} \mathrm{O}_{2}$. La congélation à $-18{ }^{\circ} \mathrm{C}$ et la distribution sont effectuées selon la méthode de Tesseyre, Divanach \& Kentouri (1978). La technique de pêche entraînant une dissociation des rotifères et de leurs œufs, nous avons défini les régimes suivants : Régime $S$ : pêché dans les Salins de Villeroy, il est constitué en nombre de 58,1 p. 100 d'œufs (139 fl) et de 41,9 p. 100 de rotifères Brachionus plicatilis $(261$ ()). Régime $M$ : pêché dans la lagune d'épuration de Marseillan composé de 84,5 p. 100 de rotifères Brachionus sp. (222 (t) et de 15,5 p. 100 d'oufs $(140$ i).

Les essais $E_{1}$ et $E_{2}$ sont effectués du 29-11-1979 au 03-01-1980 avec des densités initiales de 5,5 larves par litre dans une eau de $14,8{ }^{\circ} \mathrm{C}$ et 36 p. 1000 de salinité brassée en permanence par une aération de $25 \mathrm{ml} / \mathrm{sec}$. L'alimentation est constituée exclusivement avec le régime $S$. Les essais $E_{33}, E_{4}, E_{5}$ ont lieu du 16-01-1980 au 19-04-1980 avec des densités initiales de 23 larves/litre dans une eau stagnante durant la période prélarvaire et légèrement brassée ultérieurement de façon mécanique grâce à une pale faisant 1 tour/minute. Les animaux y reçoivent d'abord des æufs de moule $\left(E_{3}, E_{4}\right)$ ou le régime $M\left(E_{,,}\right)$jusqu'à 10 jours, puis un régime $S$ jusqu’à 44 jours 
et enfin des copépodes. Ponctuellement, des tests d'appétence sont effectués par distribution en très faible quantité d’œufs de Phallusia mammilata, de Paracentrotus lividus, de nauplii de Copépodes, de miettes de poisson et d'aliment composé (Aqualarve).

\section{Observations quotidiennes}

Leéthogramme de la larve de daurade étant inconnu, le comportement des animaux dans les bassins fait l'objet d'un suivi de 6 à 10 heures par jour (entre $9 \mathrm{~h}$ et 22 h) pendant toute la durée des essais. Le mode de nage, la dispersion dans le bac, la modification de la répartition lors de distribution d'aliment, laptitude à localiser le distributeur, l'agressivité, l'apparition du schooling et la formation de banc à l'approche de l'expérimentateur, le rhéotropisme et le phototactisme sont notés sur des fiches journalières. Les individus présentant des écarts au schéma général font l'objet d'études particulières avant d'être prélevés et disséqués.

Jusqu'à 40 jours, 3 à 30 individus par bac sont prélevés journellement, mesurée de vivo sous loupe binoculaire équipée d'un micromètre oculaire au $1 / 100^{\circ}$ de millimètre et disséqués avec des aiguilles d’entomologiste. La présence du globule lipidique, le taux de réplétion stomacale et l'état général sont notés. Le contenu du tube digestif est extrait et répertorié en nombre de proies, catégorie systématique, forme, taille et état de digestion. Ce paramètre est estimé de façon visuelle selon une échelle empirique à 4 niveaux basée sur l'état de lyse des proies :

- niveau 1 dénommé proie intacte car toutes les structures sont aisément identifiables ;

- niveau 2 dénommé début de digestion. La proie est légèrement compactée et le taux de lyse inférieur à 20 p. 100 ;

- niveau 3 dénommé stade de digestion avancé. La proie est fortement compactée et le taux de lyse est compris entre 20 et 80 p. 100 ;

- niveau 4 ou stade de la coquille vide. Le taux de lyse est supérieur à 80 p. 100 , la compaction est extrême, la proie est réduite à une enveloppe ou à une coquille transparente, fripée, contenant au maximum une ou deux gouttelettes ambrées.

Selon les jours et les bassins, les prélèvements (lots de 3 individus) sont effectués de façon à couvrir par tranches horaires toute la période comprise entre - 1 minute et +9 heures après le début de la distribution. Au-delà de 40 jours, les prélèvements de poissons sont rendus hebdomadaires.

La croissance des larves est déduite de 995 mesures effectuées depuis l'éclosion jusqu'à la taille de $27 \mathrm{~mm}$. Elle résulte des moyennes journalières de taille de tous les animaux sains disséqués auxquelles s'ajoutent celles effectuées sur des échantillons de 50 individus durant les 4 jours du stade prélarvaire et à 10, 40, 70 et 90 jours.

Jusqu'à la taille de $5 \mathrm{~mm}$, la densité en larves et le taux de survie sont estimés tous les jours par 20 prélèvements de 31 d'eau dans une cuve en plexiglas. Au-delà de cette taille, la répartition devenant hétérogène et les phénomènes d'échappement fréquents, la survie larvaire est déduite du nombre de morts récupérés par siphonage biquotidien du bac. 


\section{Observations ponctuelles}

Parallèlement aux observations précédentes, des essais ponctuels, liés à des conditions particulières ont été menés.

Les raisons de la stimulation alimentaire chez la larve de $4,5 \mathrm{~mm}$ sont recherchées par observation directe de toutes les réactions de 47 individus choisis au hasard dans le bac d'élevage et suivis individuellement de 60 à 200 secondes jusqu'à ce qu'ils disparaissent. La durée d'observation est chronométrée. Le nombre de fois où la larve s'intéresse à une proie (arrêt et observation), l'attaque, l'ingère ou la régurgite est noté et ramené à la minute. Dans chaque cas, les caractéristiques de proies inadéquates ou stimulantes sont déterminées par comparaison, sous loupe binoculaire, des particules délaissées, prélevées à la micropipette avec celles ingérées recueillies après prélèvement des larves et dissection.

Les raisons du transfert de l'activité de chasse vers des bulles sont recherchées tout au long des essais : les larves malades sont systématiquement prélevées et leur contenu stomacal est, après dissection, comparé avec celui des individus sains prélevé le même jour. La vitesse d'excrétion des bulles est déterminée après avoir isolé dans un récipient de 51,100 individus malades qui sont disséqués à raison de 5 toutes les 15 minutes.

La vitesse de digestion des Rotifères, des œufs de Rotifères et des aufs de Rotifères traités au $\mathrm{HCl} \mathrm{N} / 10$ pendant 20 minutes est estimée le 21-12-1979 chez la larve de $4,5 \mathrm{~mm}$ après avoir isolé dans des récipients de $5 \mathrm{l}$, exempts de nourriture, 3 lots de 30 larves préalablement nourries ad libitum pendant deux heures avec les régimes précités. Dans chaque cas, l'évolution du contenu stomacal est déterminée par prélèvement et dissection de 3 individus par heure.

$\mathrm{Au}$ total, nous avons disséqué 473 larves saines de 3,71 à $6 \mathrm{~mm}$ et 45 supérieures à $6 \mathrm{~mm}$ ainsi que 128 larves malades de 4,5 à $10,7 \mathrm{~mm}$ et 232 larves de 4,5 à $6,7 \mathrm{~mm}$ ayant ingéré des bulles. 2596 proies, dont 235 provenant de larves malades ont été extraites de leur tube digestif, isolées et répertoriées. 37 d'entre elles qui sont des débris animaux ou végétaux n'ont pas pu être déterminées avec précision et ont été supprimées des comptages.

\section{3. - Résultats}

\section{1. - Aspect aquacole (figure 1)}

L'élevage larvaire de Sparus auratus avec du zooplancton congelé comme unique source de nourriture, nous a permis d'amener jusqu'au stade alevin $(27 \pm 3 \mathrm{~mm})$, 1,1 p. 100 du stock des animaux des essais $E_{3}, E_{4}, E_{5}$ en 92 jours alors que tous ceux des essais $E_{1}$ et $E_{2}$ sont morts au bout d'un mois.

La phase alecithe s'est déroulée conformément à la description que nous avons faite (Kentouri \& Divanach, 1982) et l'érosion du stock $E_{1} E_{2}$ à cette période est à relier avec les perturbations comportementales dues au stress d'une aération inadéquate. La phase trophique a débuté à $3,81 \mathrm{~mm}$ avec des œufs de moule et à $4 \mathrm{~mm}$ 
avec les régimes $M$ et $S$. Dès le début, elle a présenté 4 caractéristiques limitantes particulièrement exacerbées dans les essais $E_{1} E_{2}$ : apprentissage de chasse asynchrone, sélectivité alimentaire intense tant sur le plan qualitatif que dimensionnel, transfert de l'activité de chasse sur les bulles, problème digestif. Les conséquences sont une résorption lipidique à 10 jours, une croissance négligeable jusqu'à 20 jours et même légèrement négative entre 6 et 10 jours, une mortalité chronique dans les essais $E_{1} E_{2}$ et brutale à 15 jours dans les essais $E_{3}, E_{4}, E_{5}$, une phase comportementale critique de 15 à 45 jours, un important décalibrage des tailles à 90 jours, une morphologie de juvénile à partir de $12 \mathrm{~mm}$.

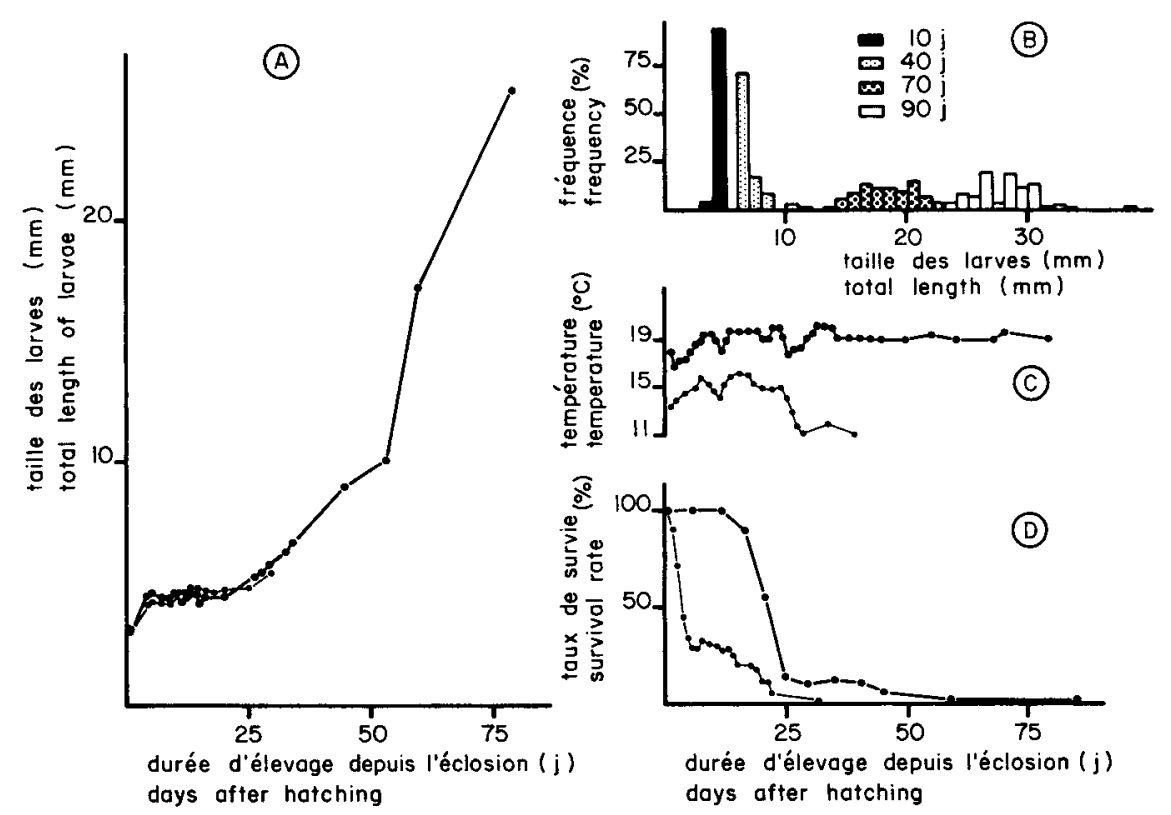

FIG. 1

FIG. 1 A : Evolution de la taille des larves au cours du temps.

Growth of the larvae during trials.

Fıg. $1 \mathrm{~B}$ : Histogrammes de répartition de la tailles des larves à 10, 40, 70, 90 jours. Distribution of total length at 10, 40,70,90 days.

Fig. $1 \mathrm{C}$ : Evolution de la température de l'eau pendant les essais. Variation of water temperature during trials.

FIG. $1 \mathrm{D}:$ Evolution du stock au cours du temps depuis l'éclosion.

Variation of survival rate after hatching.

(.) Essais $E_{1}$ et $E_{2}$. (•) Essais $E_{3}, E_{4}, E_{5}$.

(.) Trials $E_{1}$ and $E_{8}$ (๑) Trials $E_{3}, E_{4}, E_{5}$.

\section{2. - Comportement général}

De 6 à 15 jours, les larves sont dispersées de façon homogène; elles prospectent le milieu dans toutes les directions mais ne sont pas capables de localiser les points de distribution de nourriture. Leur replétion stomacale est importante. Les relations intraspécifiques sont nulles. 
Pendant la phase critique, 15 à 45 jours, soit $4,7-9 \mathrm{~mm}$ de longueur totale, la répartition spatiale devient hétérogène. Le phototropisme concentre une partie de la population dans les zones fortement éclairées; le rhéotropisme en oriente une fraction dans un périple circulaire à contre-courant; le reste se localise au centre du bac dans la tranche d'eau $0-20 \mathrm{~cm}$ ou au fond. L'extrême surface et les parois n'attirent que des individus dépigmentés à comportement anormal faisant alterner des phases stationnaires à contre-courant suivies de brefs instants de nage accélérée en position quasi verticale à l'interface eau-air avec des périodes d'hyperexcitation en un point de la paroi suivies de phases d'inertie pendant lesquelles l'animal est entraîné par le courant. Deux phénomènes d'agressivité apparaissent à la taille de $6 \mathrm{~mm}$.

a) attaque de la caudale d’un congénère éventuellement poursuivi si sa réaction de fuite est trop lente ;

b) affrontement de deux larves en position de $\mathrm{S}$ qui sobservent puis se séparent ou au contraire se détendent et s'entrechoquent.

De 45 à 92 jours ( 9 à $27 \mathrm{~mm}$ ) le comportement se modifie à nouveau. La dispersion redevient homogène mais une stratification par classe de taille s'établit, les gros individus se localisent près $d u$ fond sur lequel ils commencent à manger. L'agressivité disparaît, les relations intraspécifiques se limitent à des raisons alimentaires :

a) synchronisation des réactions d'approche lors de la distribution d'aliment;

b) compétition et poursuite de 2 à 5 individus pour une particule alimentaire de grande taille. L'activité de prospection est intense notamment au niveau du distributeur. L'animal semble adapté à la nourriture et au milieu, il est très confiant et vient même manger dans la main.

\section{3. - L'apprentissage de chasse}

Même avec du zooplancton congelé, l'entrée dans la phase trophique passe par une période d'apprentissage identique à celle décrite par Kentouri \& Divanach (1982). Du $4^{\circ}$ au $7^{\circ}$ jour après l'éclosion, la séquence entre la découverte de la proie et lattaque évolue vers un arc réflexe rapide, les postures «prédétente » en virgule font place aux formes classiques en $\mathrm{S}$, la fréquence des échecs diminue. L'ingestion d'aliment résulte d'une expérience réussie car la replétion stomacale est d'emblée importante ou alors le tube digestif reste vide. Les aufs de moule sont attaqués avec succès entre 4 et 5 jours, ceux de rotifères à 6 jours, les rotifères inférieures à 200 $\imath$ à 7 jours. Les grosses femelles parthénogénétiques et les copépodes induisent des réactions de positionnement mais sont systématiquement manqués ou délaissés. Ces dernières proies entraînent une désynchronisation de prise de nourriture initiale dans la population (Fig. 2 B), un transfert de l'activité de chasse sur des bulles ou des œufs de rotifères, une mortalité rapide.

\section{4. - Sélectivité alimentaire}

Elle est visualisée dans les figures 2 et 3 ainsi que les tableaux 1 à 4 et se résume de la façon suivante:

a) dès les premiers jours d’alimentation, la larve présente deux traits de compor- 

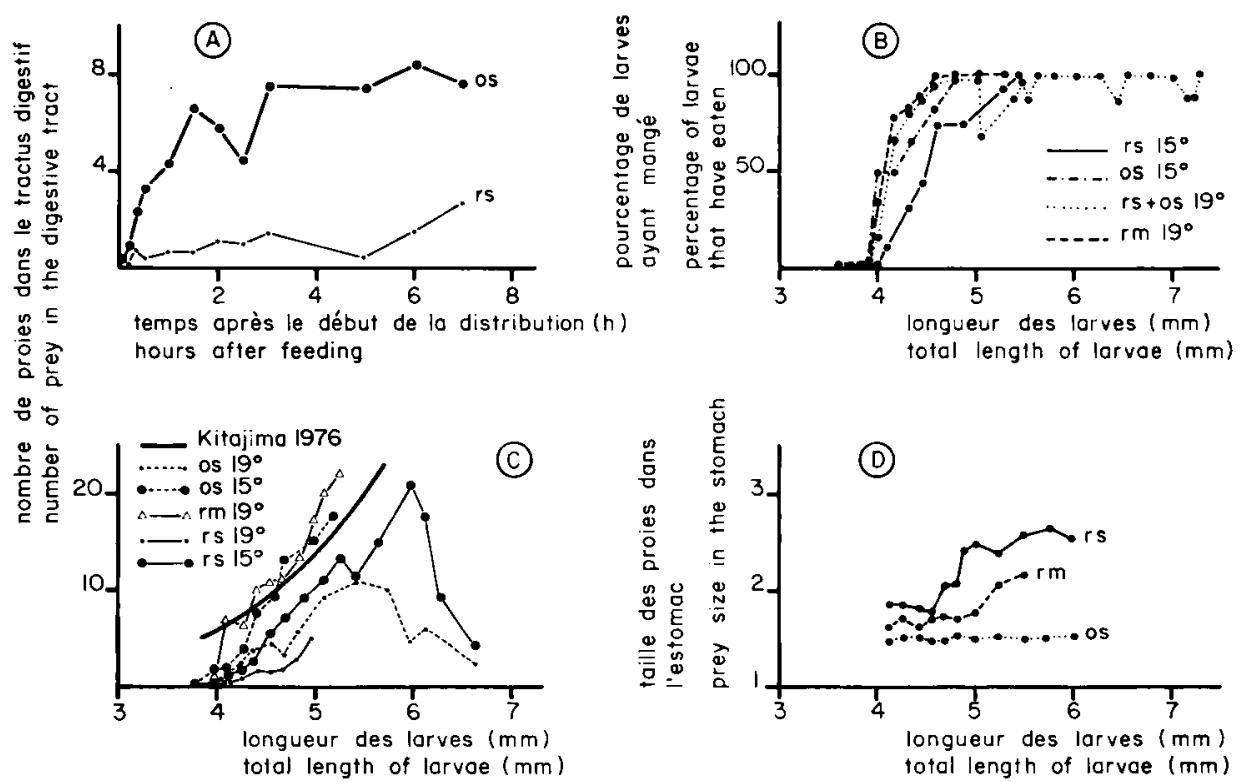

FiG. 2

Fig. 2 A : Evolution au cours du temps du nombre de proies dans le tractus digestif chez les larves de 4,4 mm (distribution en continu de l'aliment).

Number of prey in the digestive tract of $4.4 \mathrm{~mm}$ larvae after feeding.

Fig. 2 B : Evolution du pourcentage de larves ayant mangé 2 heures après distribution, en fonction de leur taille.

Relation between the rate of larvae having eaten 2 hours after feeding and their total length.

Fik. $2 \mathrm{C}$ : Evolution du nombre de proies dans le tractus digestif des larves en fonction de leur taille (prélèvements effectués 2 heures après distribution).

Relation between the number of prey in the digestive tract of the larvae 2 hours after feeding and their total length.

FIG. 2 D : Evolution de la taille des proies dans l'estomac en fonction de la taille des larves. Relation between the prey length in the stomach and the total length of the larvae.

RS : Rotifères des salins. RM : Rotifères de Marseillan. OS : Eufs de Rotifères des salins. $15^{\circ} \mathrm{C}$ et $19^{\circ} \mathrm{C}$ : Température de l'essai.

RS : Large Rotifers from Villeroy. RM : Small Rotifers from Marseillan. $O S$ : Eggs of Rotifers. $15^{\circ} \mathrm{C}$ and $19^{\circ} \mathrm{C}$ : Temperature of trials.

tement qui aboutissent à la non ingestion des aliments : l'abandon après observation et la régurgitation après attaque. Leur fréquence d'apparition dépend du moment de distribution de l'aliment (tableau 1) et évolue dans le temps. 84 p. 100 des proies observées sont délaissées et 62 p. 100 de celles attaquées sont régurgitées par des larves de 4,1 à $4,4 \mathrm{~mm}$ de taille. Pendant la période critique, la sélection visuelle prend une importance si grande et si rapide que les comptages sont perturbés. Nous estimons que 98 p. 100 des proies sont délaissées sur simple observation. Par opposition, les régurgitations ne représentent plus que 20 p. 100 des attaques. Au-delà de 
$9 \mathrm{~mm}$, le phénomène s'inverse à nouveau : les larves semblent en permanence affamées et «goûtent» à tout ce qu'elles rencontrent. Les proies ne sont plus régurgitées et abandonnées, mais reprises de 5 à 10 fois sous les angles parfois différents, entièrement englouties ou seulement pincées entre les mâchoires, avant d'être avalées ou délaissées.
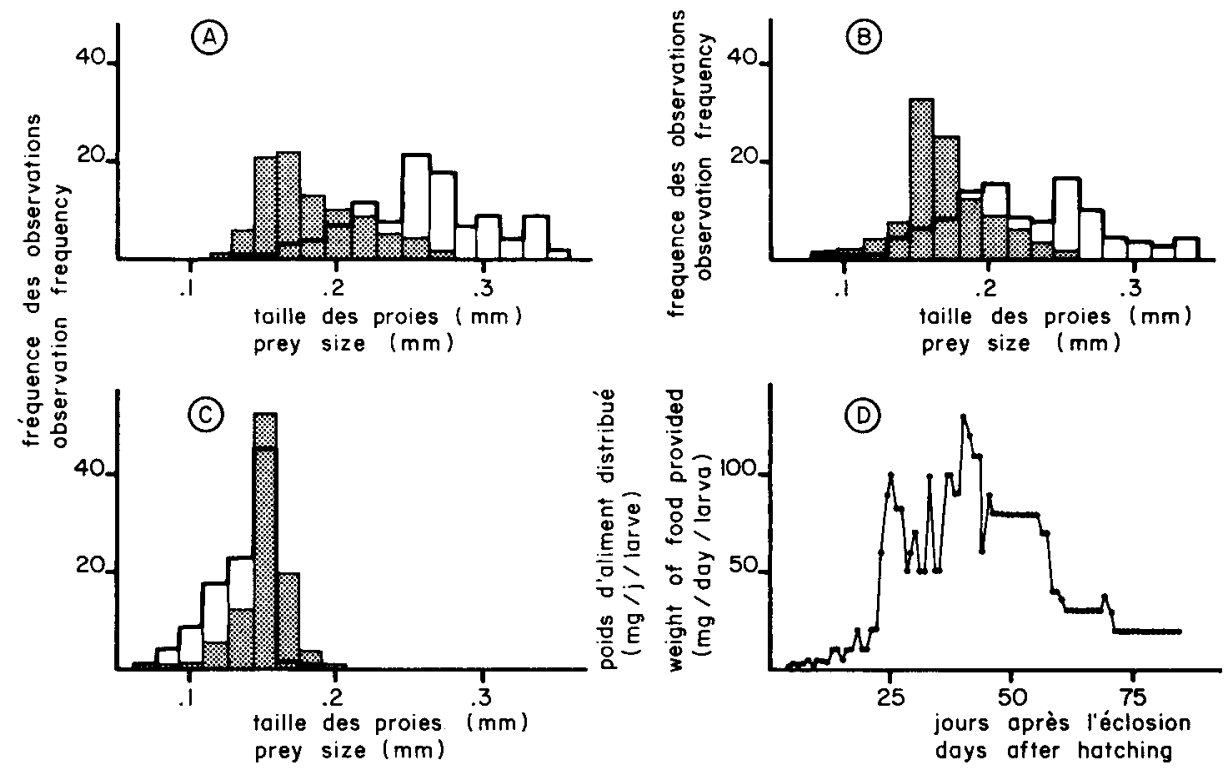

FiG. 3

FIG. $3 \mathrm{ABC}$ : Comparaison de la taille des proies distribuées larves de 4,1 à $4.4 \mathrm{~mm}$.

Comparison of the size of the prey offered $\square$ and ingested by larvae of 4.1 to $4.4 \mathrm{~mm}$.

A : Rotifères des salins. B : Eufs de Rotifères des salins. C : Rotifères de Marseillan. A : Large Rotifers from Villeroy. B : Eggs of Rotifers. C: Small Rotifers from Marseillan.

FIG. $3 \mathrm{D}$ : Evolution de la quantité d'aliment distribuée pendant la période d'élevage sur Rotiferes (.) et sur Copépodes $(\bullet)$.

Daily amount of food given to a larva during the trials with Rotifers (.) and Copepods $(\bullet)$.

L'analyse microscopique des particules ayant suscité l'attention des larves mais n'ayant pas conduit à l'attaque montre (tableau 2) que leur profil type comporte une composante qualitative ou dimensionnelle instantanément reconnue. Les catégories indigestes (détritus, flocons bactériens, scories), dénaturées (agrégats de rotifères, rotifères fripés ou vides) ou supérieures à 200 ॥ sont prépondérantes. Seuls 19 p. 100 des rotifères délaissés présentaient des critères anatomiques des proies normalement ingérées. Par opposition aux précédentes, les proies régurgitées semblent adéquates. Nous supposons que leur identification comporte une composante organoleptique. 


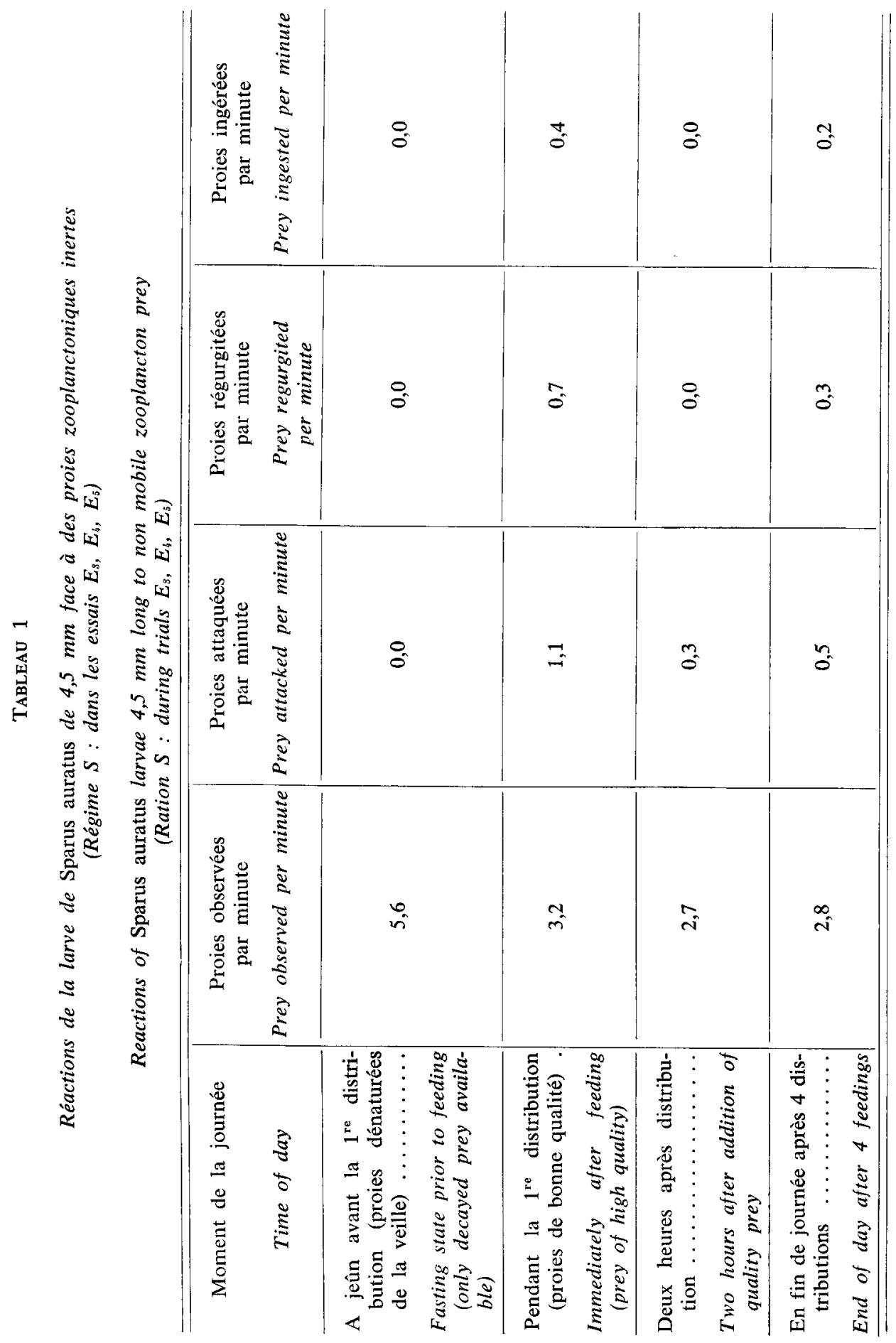




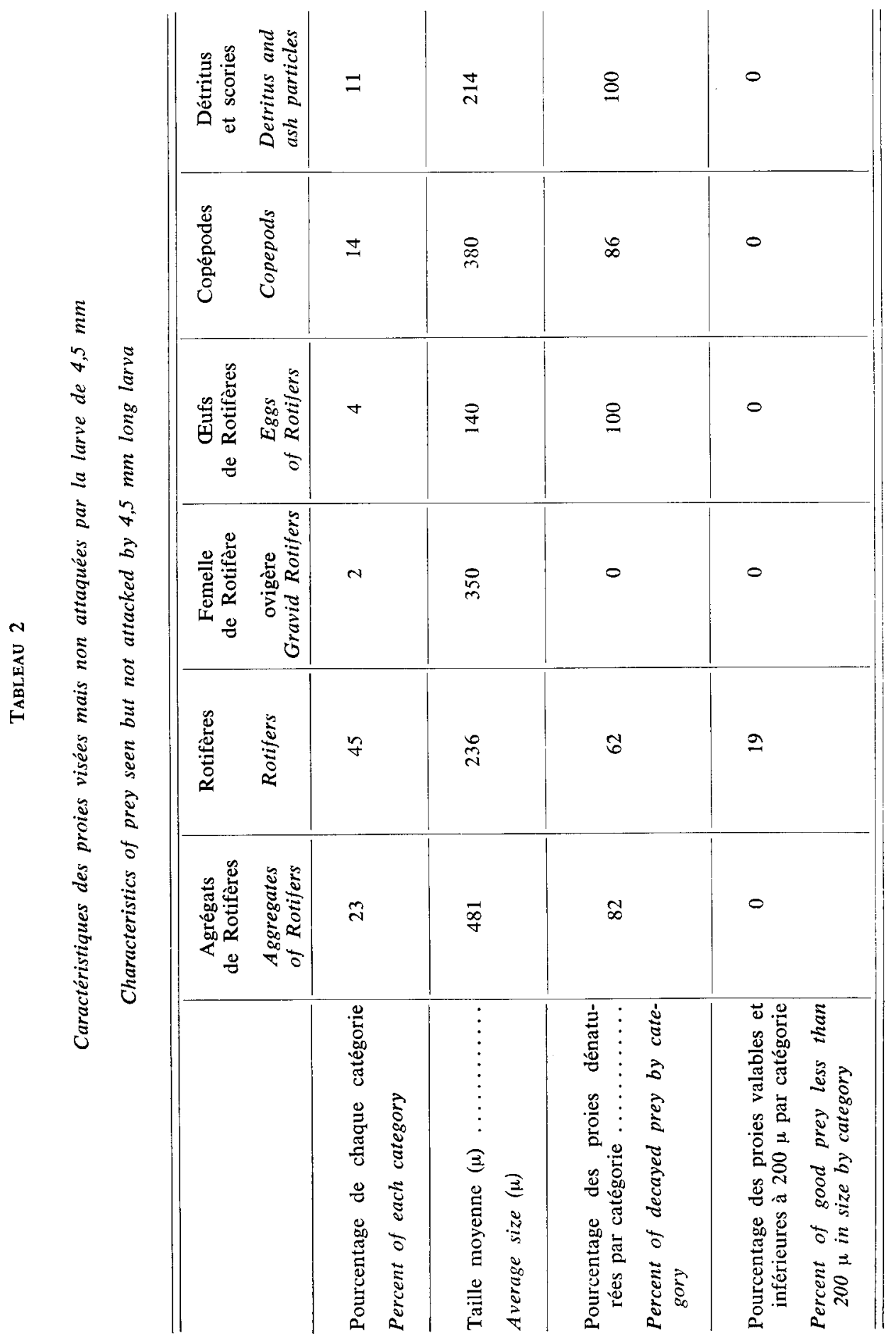


La juxtaposition des 2 comportements sélectifs se traduit par une importante perte de temps de prospection. Dans le meilleur des cas, seuls 14 p. 100 des proies observées sont ingérées, ce qui explique la durée de replétion stomacale (fig. $2 \mathrm{~A}$ ).

b) le nombre de proies dans le tractus digestif et leurs caractéristiques évoluent avec la taille des larves (fig. $2 \mathrm{C}$ et $\mathrm{D}$ ). Les animaux de 4,1 à $4,4 \mathrm{~mm}$ sélectionnent les proies comprises entre 130 et $200 \mu$. Ces classes de tailles très peu représentées dans les régimes de rotifères distribués, se retrouvent avec des fréquences élevées (valeurs modales) dans le contenu stomacal (fig. $3 \mathrm{~A}, \mathrm{~B}, \mathrm{C}$, tableau 3). Leur nombre limité notamment dans le régime $S$, peut expliquer le transfert de l'activité de chasse vers les cufs de rotifères dans les essais $E_{1}$ et $E_{2}$ (tableau 4).

\section{TABleau 3}

Comparaison de la fréquence d'observation de certaines classes de taille dans la ration distribuée et le contenu stomacal

Comparison of the frequency of prey size class ingested relative to size class available

\begin{tabular}{l|c|c|c}
\hline \hline & $\begin{array}{l}\text { Distribués } \\
\text { Available }\end{array}$ & $\begin{array}{c}\text { Ingérés } \\
\text { Ingested }\end{array}$ \\
\hline $\begin{array}{l}\text { Rotifères des Salins. Inférieurs à } 186 \mu \ldots \\
\text { Rotifers from Villeroy less than } 186 \mu\end{array}$ & $5,8 \%$ & $60,4 \%$ \\
\hline $\begin{array}{l}\text { Rotifères de Marseillan. Inférieurs à } 186 \mu \ldots \ldots \\
\text { Rotifers from Marseillan less than } 186 \mu\end{array}$ & $32,7 \%$ & $76,9 \%$ \\
\hline \hline
\end{tabular}

Tableav 4

Comparaison du pourcentage d'œufs de Rotifères dans la fraction distribuée et la fraction ingérée par les larves de différentes tailles

The percent of Rotifer eggs ingested by larvae of different sizes relative to the number of eggs available

\begin{tabular}{|c|c|c|c|c|c|c|c|c|}
\hline & \multirow[t]{2}{*}{$\begin{array}{c}\text { Fraction } \\
\text { distribuée } \\
\text { Available }\end{array}$} & \multicolumn{7}{|c|}{$\begin{array}{c}\text { Fraction ingérée en fonction de la taille des larves (mm) } \\
\text { Ingested relative to total length of larvae }\end{array}$} \\
\hline & & 4,1 & 4,29 & 4,43 & 4,57 & 4,71 & 4,86 & 5 \\
\hline $\begin{array}{l}\text { Régime } \mathrm{S} . \cdots \\
\text { Ration } S\end{array}$ & 58,1 & 80 & 71 & 54 & 45 & 31 & 23 & 17 \\
\hline $\begin{array}{l}\text { Régime } \mathrm{M} \ldots \\
\text { Ration } M\end{array}$ & 15,5 & 6 & 12 & 4 & 9 & 15 & 7 & 2 \\
\hline
\end{tabular}


Au-delà de 4,4 mm, les larves commencent à ingérer des proies de plus grande taille, mais ce n'est qu'à partir de $4,7 \mathrm{~mm}$ qu'elles attaquent avec succès tous les rotifères du régime $S$. Parallèlement, leur intérêt pour les petites tailles diminue. Les œufs de moules sont délaissés vers $4,1 \mathrm{~mm}$, ceux de rotifères vers $5,5 \mathrm{~mm}$.

De 6 à $9 \mathrm{~mm}$, les proies ingérées mesurent de 200 à $500 \mu$. Malgré le pourcentage élevé de ces classes de taille dans la ration, les larves présentent une sélectivité alimentaire intense que nous n'avons pas réussi à expliquer. Elles boudent les régimes $\mathbf{M}$ et $\mathbf{S}$, refusent totalement les copépodes, les miettes de poisson, le granulé, les œufs de Phallusia mammilata et de Paracentrotus lividus. Elles ne sont pas plus attirées par les couleurs rouges (plancton cuit) que brunes (plancton cru), mais sont fortement stimulées par les bulles. Elles semblent avoir une vision défectueuse car elles réagissent peu aux mouvements de l'observateur et la prise de nourriture est tardive dans la matinée (l'éclairage artificiel est nécessaire). Les contenus stomacaux diminuent et la résistance au jeûne devient inférieure à 24 heures. Cette période critique nous a amenés à effectuer des distributions d'aliment abusives (fig. $3 \mathrm{D}$ ) avec des fréquences très rapprochées ( 9 repas dans la journée et distribution continue de $21 \mathrm{~h}$ à $9 \mathrm{~h}$ ) dont la presque totalité est évacuée par siphonage. Malgré cela une mortalité chronique, précédée d'une dépigmentation et d'une montée dans les couches superficielles, s'instaure. 17 p. 100 de ces animaux n'ont aucun symptôme clinique mais quelques-uns en présentent plus d'un : hydropisie ( 2 p. 100), parois intestinales fines et fragiles ( 7 p. 100), état de maigreur extrême (14 p. 100), vessie natatoire hyperdéveloppée (17 p. 100), bulle d'air dans l'estomac (19 p. 100), tube digestif vide (21 p. 100).

Au-delà de $9 \mathrm{~mm}$, l'état général s'améliore rapidement. La sélectivité alimentaire devient minime. En permanence affamées, les larves ingurgitent indifféremment copépodes, agrégats de rotifères, daphnies, miettes de poisson ou même granulé. Elles se jettent dans les «nuages alimentaires» de petites tailles (rotifères, petits copépodes) qu'elles filtrent frénétiquement ou s'attaquent à des proies trop grosses qu'elles essaient de déchiqueter. Elles évoluent souvent près du fond pour manger et commencent vers $20 \mathrm{~mm}$ à acquérir un nouveau mode de prospection alimentaire benthique qui consiste à trier les proies mélangées aux détritus par le biais d'une bouchée initiale non sélective et d'une régurgitation violente qui amène par effet de souffle une dispersion des particules et leur remise en suspension locale. Les pertes d'aliment par sédimentation sont ainsi minimisées.

\section{5. - Maladie de la bulle}

Dès les premiers jours d'alimentation, les larves s'intéressent aux bulles issues de la photosynthèse ou de l'aération. Jusqu'à $6 \mathrm{~mm}$, elles les avalent et déséquilibrées s'accumulent en surface alors qu'au-delà, elles les régurgitent rapidement. Les individus les plus touchés sont ceux de 4 à $4,5 \mathrm{~mm}$ (fig. $4 \mathrm{~A}$ ).

Dans 62 p. 100 des cas, ils n'attaquent qu'une seule bulle mais nous en avons dénombrées jusqu'à 8 dans leur tube digestif (fig. $4 \mathrm{D}$ ). La taille des bulles ingérées varie de 50 à $350 \mu$. Les valeurs modales et moyennes (respectivement 150 à $177 \mu$ ), sont très proches de celles de la taille des proies sélectionnées par ces mêmes larves (fig. 4 B). L'ingestion de bulles n'est pas mortelle car les larves arrivent à les excréter assez vite (fig. $4 \mathrm{E}$ ), mais elle entraîne une immobilisation du tractus digestif pendant 

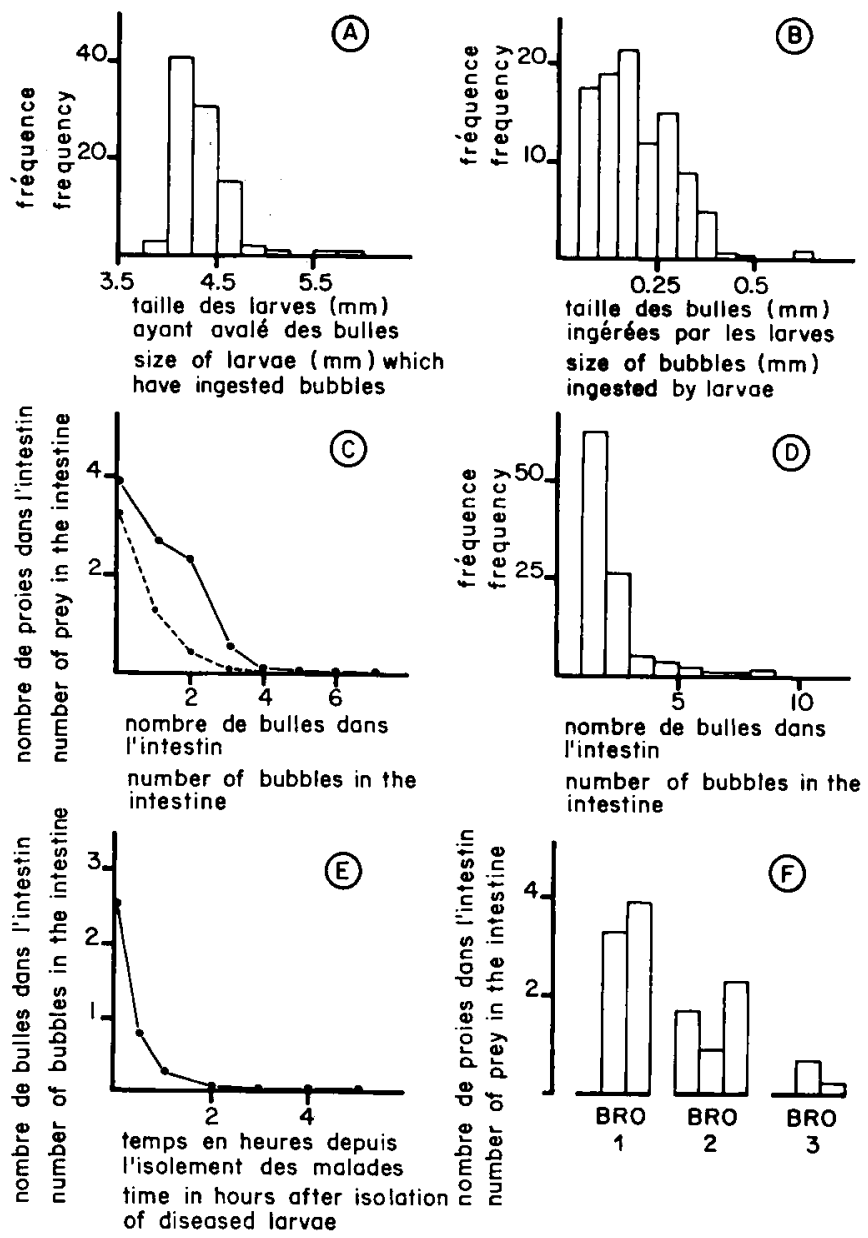

FIg. 4

FIG. $4 \mathrm{~A}$ : Relation entre la taille des larves et la fréquence d'observation de la maladie de la bulle.

Relationship between total length of larvae and the frequency of gas bubble disease.

FIG. 4 B : Spectre de distribution de la taille des bulles ingérées par les larves.

Histogram of size distribution of bubbles ingested by larvae.

FIG. $4 \mathrm{C}$ : Relation entre le nombre de bulles dans l'intestin et le nombre de proies : (......) Rotifères, (-) oufs de Rotifères.

Relation between the number of bubbles in the intestin and the number of prey : (......) Rotifers, (-) eggs of Rotifers.

FIG. $4 \mathrm{D}$ : Histogramme de répartition du nombre de bulles ingérées par larve. Histogram of the distribution of number of bubbles ingested per larva.

FIg. $4 \mathrm{E}$ : Courbe d'excrétion des bulles au cours du temps après isolement des malades $\left(T^{\circ}: 20^{\circ} \mathrm{C}\right)$.

Curve of excretion of bubbles at $20^{\circ} \mathrm{C}$ after isolation of diseased larvae.

Fic. $4 \mathrm{~F}$ : Contenu stomacal moyen d'une larve saine (1), d'une larve ayant ingéré des bulles (2) et d'une larve venant d'excréter les bulles (3).

Stomach contents of (1) healthy larva, (2) larva which has ingested bubbles and, (3) larva which has excreted bubbles.

$B$ : Bulles ; $\mathbf{R}$ : Rotifères; $\mathbf{O}$ : CEufs de Rotifères.

$B:$ Bubbles ; $R:$ Rotifers; $O$ : Eggs of Rotifers. 
environ $1 \mathrm{~h} 30$ et une réduction du nombre de proies dans l'estomac d'autant plus importante que le nombre de bulles est élevé (fig. $4 \mathrm{C}$ ). L'encombrement digestif et le déséquilibre occasionné par la flottabilité peuvent être responsables de ce phénomène. Le processus d'élimination des bulles semble lié à une modification de leur tension superficielle, car elles éclatent sitôt arrivées en surface. Par ailleurs, il met en jeu un mécanisme musculaire intense qui amène une purge du tractus digestif (fig. $4 \mathrm{~F}$ ).
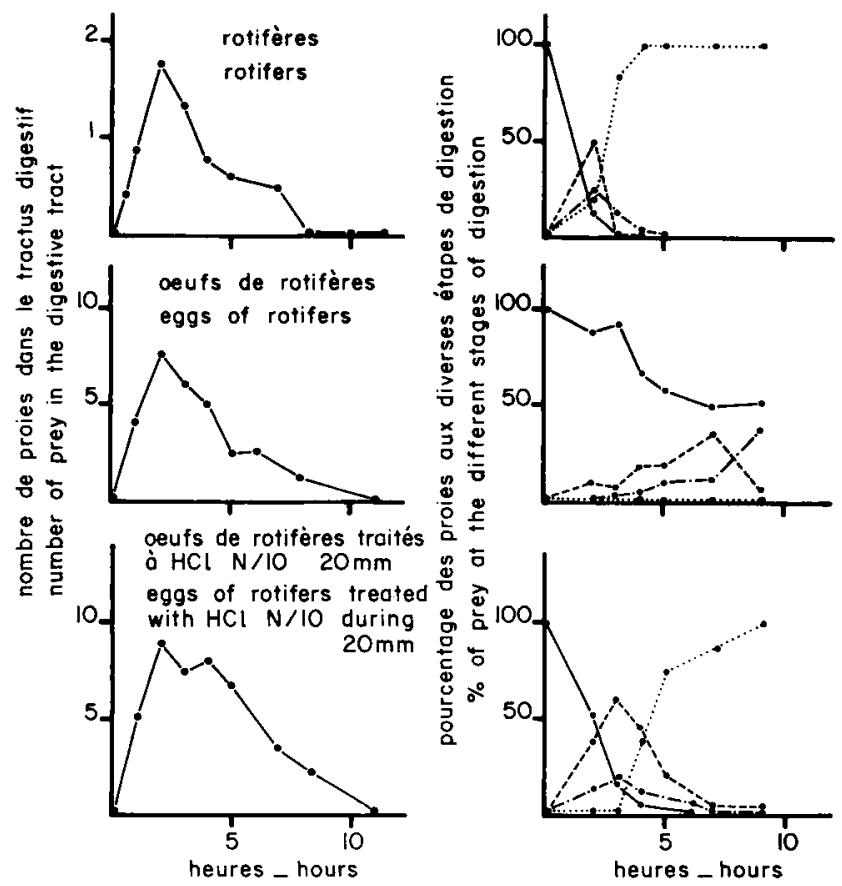

Fig. 5

Evolution au cours du temps du nombre et de l'état de digestion des proies dans le tractus digestif de larves de daurades de $4,5 \mathrm{~mm}$, nourries ad libitum, puis mises en eau exempte de nourriture $\left(20^{\circ} \mathrm{C}\right)$ all bout de 2 heures

Development over time of the number and stage of digestion of prey in the digestive tract of $4.5 \mathrm{~mm}$ long. Sea bream larvae fed ad libitum during two hours and then transfered in water with no prey

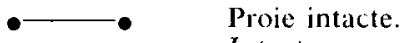

Intact prey.

Proie en voie de digestion $(<20$ p. 100).

Prey in initial stage of digestion $(<20 \mathrm{p} .100)$.

- - - Proie dont la digestion est avancée (20 à 80 p. 100).

Prey well digested (20 to $80 \mathrm{p}$. 100).

-..... Proie réduite à une enveloppe ou une coquille vide.

Prey reduced to an empty shell. 


\section{6. - Réplétion et déplétion stomacales}

Cette étude montre que les larves s'alimentent dès la distribution de nourriture $(\mathrm{Vi}=0,85$ rotifère/heure $+3,5$ aufs de rotifère/heure pour la larve de $4,5 \mathrm{~mm}$ à jeûn) et que sitôt mises en eau propre, elles commencent à excréter des boulettes fécales de 170 à $420 \mu$ de long sur 140 à $170 \mu$ de large qui contiennent de 5 à 20 enveloppes de rotifères compactées ainsi que les coquilles d'œufs vides. Les œufs non digérés, sont expulsés par petites grappes de 2 à 5 .

\section{7. - Digestibilité des aliments}

Avec notre méthode, la digestibilité des œufs de moules est difficile à estimer car dans le tube digestif ces proies s'amalgament en un cordon indissociable, mais elle semble incomplète. L'état de digestion des autres proies est aisé à estimer car elles conservent leur identité. Chez la larve de $4,5 \mathrm{~mm}$ rassasiée, puis mise en eau propre, les rotifères entrent en digestion quelques dizaines de minutes après l'ingestion et leurs enveloppes vides sont toutes excrétées au bout de 6 heures (fig. $5 \mathrm{~A}$ ). Par contre, avec une alimentation en continu, 38 p. 100 de ces proies sont excrétées sous forme de pseudo fèces à un stade de digestion compris entre 20 et 80 p. 100 (tableau 5). Avec les aufs de rotifères, le phénomène est bien différent : 50 à 60 p. 100 d'entre eux, suivant le mode de distribution (ponctuel ou continu), sont totalement réfractaires à toute attaque enzymatique. Malgré un transit digestif de 9 heures, seuls 15 p. 100 atteignent le stade de coquille vide et 20 p. 100 sont excrétés alors que leur état de digestion n'est que de 20 à 80 p. 100 (fig. 5 B, tableau 5).

\section{TABLEAU 5}

Comparaison de l'état de digestion des Rotifères et de leurs aufs dans le rectum et le tube digestif (en pourcentage des proies) en alimentation continue

Comparison of the stage of digestion of Rotifers and their eggs in the rectum and digestive tract (percent of prey) continuous feeding

\begin{tabular}{l|c|c|c|c|c|c|c|c}
\hline \hline & \multicolumn{3}{|c|}{$\begin{array}{c}\text { Eufs de Rotifères } \\
\text { Eggs of Rotifers }\end{array}$} & \multicolumn{3}{|c}{$\begin{array}{c}\text { Rotifères } \\
\text { Rotifers }\end{array}$} \\
\hline $\begin{array}{l}\text { Tube digestif .... } \\
\text { Digestive tract }\end{array}$ & 59 & 16 & 12 & 14 & 3 & 15 & 53 & 29 \\
\hline Rectum ........ & 63 & 3 & 19 & 14 & 1 & 1 & 38 & 60 \\
\hline
\end{tabular}

I : Proie intacte; E : Proie en voie de digestion $(<20 \%) ; \mathrm{D}$ : Proie dont la digestion est avancée $(20$ à $80 \%) ; \mathrm{V}$ : Proie réduite à une coquille vide. Etude faite sur 1680 proies dont 1276 dans le tube digestif.

I : Intact prey; E : Prey in initial stage of digestion $(<20 \%) ; D:$ Prey well degraded $(20$ to $80 \%) ; V$ : Prey reduced to an empty cuticle. Study made over 1680 prey from wich 1276 were in the digestive tract. 
L'hydrolyse acide des œufs de rotifères $(\mathrm{HCl} \mathrm{N} / 1020$ minutes) augmente la digestibilité du produit à condition que le transit intestinal dépasse 5 heures (fig. $5 \mathrm{C}$ ), c'est-à-dire qu'on limite la production de pseudo fèces par des distributions ponctuelles.

Les copépodes présentent des états et des vitesses de digestion identiques à ceux des rotifères.

\section{4. - Discussion}

L'utilisation des proies zooplanctoniques congelées pour l'élevage des premiers stades larvaires de Sparus auratus n`avait jusquà présent pas été signalée. Les résultats obtenus ici liés à ceux de Kentouri $(1980,1981)$ et Kentouri, Divanach \& Cantou (1979) sur les larves de Dicentrarchus labrax et les post-larves de Diplodus sargus, Diplodus vulgaris, Mugil cephalus, Penaeus japonicus, Anguilla anguilla précisent les espoirs que l'on peut fonder sur l'alimentation larvaire avec proies inertes.

Les taux de survie à 3 mois enregistrés lors des essais $E_{3}, E_{4}, E_{\bar{j}}(1,1$ pour 100) sont du même ordre de grandeur que ceux obtenus par BarNabe (1976) et RAmos (1978) avec du zooplancton vivant. Le retard de croissance par rapport aux animaux nourris avec la séquence traditionnelle (vivante) est comparable à celui observé par Kentouri (1978) sur le loup alimenté avec du zooplancton congelé. Comme l'a obtenu Kitajima (1979) sur Pagrus major et à condition que la première alimentation soit de très petite taille (œufs de moules, petits rotifères), nous trouvons un taux de survie proche de 100 p. 100 jusquà 15 jours, puis une phase critique qui dure de 15 à 45 jours pendant laquelle la mortalité (voisine de 95 p. 100) est supérieure à celle signalée par cet auteur. Les phases post-larvaires et juvéniles ont une survie supérieure à 80 p. 100 .

La période critique a retenu notre attention et nous avons essayé de la limiter par une surveillance constante, des distributions ad libitum d'aliments (atteignant $100 \mathrm{mg} /$ larve par jour) toutes les 90 minutes, un important renouvellement d'eau et un nettoyage biquotidien du fond. Ce mode opératoire réduit les mortalités mais est incompatible avec un élevage industriel dans l'état actuel des techniques aquacoles.

Les deux principaux facteurs limitants sont dordre comportementaux et digestifs. La préférence alimentaire des premiers stades larvaires pour les proies inférieures à 200 \& peut être recherchée dans des raisons morphologiques. Houde (1973), IIzAwa (1980) ont montré la relation qui existe entre la taille des proies ingérées et l'ouverture de la bouche. Il est possible que l'ingestion préférentielle d'œufs de rotifères et l'attrait représenté par les bulles malgré des densités en proies élevées $(5$ à $20 / \mathrm{cc})$ soient des choix effectués sur un critère de taille adéquate.

L'apparition simultanée des activités trophiques et du comportement sélectif chez les larves enseigne lattention que l'aquaculteur doit porter à la qualité de l'aliment initial car la réussite de l'élevage peut en dépendre. Dès son plus jeune âge, la larve est capable de jauger la valeur dune proie de deux façons : a priori sur simple observation et a posteriori par rejet après préhension. Les attitudes similaires ont été trouvées (Kentouri, 1981) chez les post-larves de Gobius sp. et de Trigla 
corax. Le facteur conditionnant la régurgitation pourrait être d'origine organoleptique car chez les larves de loup, Kentouri (1978) a noté la diminution de l'appétence des proies zooplanctoniques congelées ayant séjourné plus de 4 heures dans l'eau. Le rôle de la vision dans les comportements "a priori » est indiscutable car les scories, les détritus, les flocons bactériens sont fréquemment l'objet d'observations mais rarement d'attaques. Une mémoire visuelle des proies inadéquates fondée sur une expérience malheureuse (attaque suivie de régurgitation) semble être à l'origine du phénomène.

La non-digestibilité de certains aliments est connue. Elle est liée à l'absence de matériel enzymatique adaptée et/ou à la présence de composés protecteurs (thèque, coquille) difficilement dégradables. RaBE, Echelle \& Schlichting (1973) ont constaté la viabilité de certaines algues dans le tractus digestif de deux cyprinodontidés. Barnabe (1976) a observé que les cufs d'Artemia peuvent être ingérés mais non digérés par les larves de loup. Irzawa (Communication personnelle) a remarqué que les œufs de rotifère Brachionus plicatilis ingérés en même temps que les femelles parthénogénétiques ne sont pas digérés par la larve de Fugu niphobles.

Les conséquences immédiates de l'ingestion d’œufs de rotifères par les jeunes larves sont :

a) une augmentation de la durée du transit intestinal de 3 heures;

b) un encombrement du tube digestif donnant limpression macroscopique d'un bol alimentaire important mais qui est démenti par un comportement affamé et un transfert de l'activité de chasse vers les bulles. Cette forme de « jeûne physiologique» pourrait être à l'origine de la précocité de la résorption lipidique et de l'acuité de la phase critique lors de la métamorphose.

\title{
5. - Conclusion
}

Les résultats obtenus mettent en évidence le comportement des larves élevées sur nourriture planctonique congelée. Ils ont permis de comprendre une des causes de mortalité qui survient chez les larves de daurade, au cours de l'élevage. Il devient maintenant indispensable de comparer les résultats avec ceux obtenus en élevage à partir de nourriture constituée de proies vivantes.

Accepté pour publication en aô̂t 1981 .

\author{
Summary \\ Food preferences and behaviour of Sea Bream larvae \\ (Sparus auratus) fed with frozen zooplankton
}

A total of 5 culture trials were made in 570 l circular tanks. Sea Bream larvae were fed with frozen zooplankton from the "mouth opening stage " until 92 days of age. Survival rates and growth are reported in figure 1. When the first feeding was composed of large sized Brachionus plicatilis $(261 \pm 47 \mu)$ and/or of Rotifers eggs, the survival rate 
was null. On the other hand, when small sized Rotifers and/or Mytilus galloprovincialis eggs were given first, results were comparable to those obtained by BarNaBE (1976) and Ramos (1978) using live feed.

Larvae began to eat earlier when fed with small sized preys (fig. 2 B) and up to $4.5 \mathrm{~mm}$ long they selectively choosed preys smaller than $200 \mu$ (fig. $2 \mathrm{D}$ and fig. 3 ). Rotifers were digested in less than 2 hours (fig. $5 \mathrm{~A}$, table 5), but when small ones were not available, larvae ate Rotifer eggs (tabl. 3 and 4) which were not digested, or bubbles of adequate size. Characteristics of this behaviour are reported in fig. 4.

Excretion of ingested bubbles occurred in less than $2 \mathrm{hrs}$ (fig. $4 \mathrm{E}$ ) resulting in a purge of the digestive tract (fig. $4 \mathrm{~F}$ ). Two mechanisms of prey selection are described (tables 1 to 4) : visual selection and regurgitation, the former being more frequent.

These behavioural patterns might explain one of the causes of mortality during larval culture of Sparus auratus.

\section{Références bibliographiques}

Arias A., Pascual E., 1979. Pilot scale production of sea bream Sparus auratus. Fry Paper presented to the Mariculture committee at the 67th statuory Meeting of I.C.E.S. Warsaw, Poland, 1-10 oct. 1979.

Barnabe G., 1976 a. Rapport technique sur la ponte induite et l'élevage des larves du loup Dicentrarchus labrax (L.) et de la daurade Sparus auratus (L). Stud. Rev., C.G.P.M., F.A.O. éd. (55), 63-116.

Barnabe G., 1976 b. Contribution à la connaissance de la biologie du loup Dicentrarchus labrax (L.) (Poisson serranidae) de la région de Sète. Thèse de doctorat ès-science, Univ. Sci. et Techni., Languedoc, $426 \mathrm{p}$.

Barnabe G., 1979. Utilisation des chaînes alimentaires naturelles et du recyclage des eaux usées dans la production à grande échelle de juvéniles pour l'aquaculture. Publication du C.N.E.X.O., Actes de colloques, 7, 221-238.

C.G.P.M., 1980. Rapport de la $4^{*}$ session du Copraq, Barcelone, Espagne, 16-19 octobre 1979. Rapport F.A.O., 232, 35 p.

Divanach P., Barnabe G., Jullian J.F., 1980. Pompe à plancton. Le marché de linnovation, $\mathbf{4 1}$, p. 16.

Fabre: J.F., 1979. Les techniques de production de masse d'alevins de daurade Pagrus major au Japon. Publication de l'Association pour le Développement de l'Aquaculture, 7. $37 \mathrm{p}$.

Fukuhara O., 1974. The influence of initial delay of feeding on survival, growth and development of the red sea bream Chrysophrys major. Bull. Nansei Reg. fish Res. Lab., 7, 19-29.

Fukusho K., Hara O., Iwamoto H., 1977. Estimation of daily amount of the Rotifer Brachionus plicatilis fed by a larva of red sea brean Pagrus major during mass rearing using large scale outdoor tanks. Bull. Nagasaki Prefect. inst. fish, 3, 41-47.

GIRIN M., 1979. Méthode de production des juvéniles chez trois poissons marins, le bar, la sole et le turbot. Publications du C.N.E.X.O. Rapports scientifiques et techniques, 39 , $202 \mathrm{p}$.

Houde E.D., 1973. Some recent advances and unsolved problems in the culture of marine fish larvae. Proc. World Mariculture society, 3. 83-112.

IIzawa M., 1980. Relation entre les dimensions de la bouche des larves du loup Dicentrarchus labrax et la taille des proies. Rapport D.E.A. Univ. Sci. Techn. Languedoc, Montpellier, $15 \mathrm{p}$.

Kentouri M., 1978. Contribution à l'étude de l'alimentation des larves de loup Dicentrarchus labrax L. à l'aide d'organismes de zooplancton congelés. Thèse doctorat 3* cycle, Univ. Aix-Marseille II, 117 p. 
Kéntovrı M., 1980. Elevage des larves de loup Dicentrarchus labrax L. à l'aide d'organismes du zooplancton congelés. Résultats préliminaires. Aquaculture, 21, 171-180.

Kentouri M., 1981. Données préliminaires sur les facultés d'adaptation à un aliment inerte (zooplancton congelé) des post-larves de 11 espèces de poissons et crustacés marins. Aquaculture, 23, 73-82.

Kintourı M., Divanach P., 1982. Différences et similitudes dans la genèse des comportements locomoteur et trophique des stades prélarvaires de Sparus auratus, Diplodus vulgaris et Diplodus sargus. Aquaculture (sous presse).

Kentouri M., Divanach P., Cantou M., 1979. Données préliminaires sur le comportement, la croissance et la survie du sar Diplodus sargus en élevage. Communication présentée à la $4^{\prime}$ session du C.O.P.R.A.Q.. Barcelone, 16-19 octobre 1979, 24 p.

Kitajima C., 1976. Amount of the copepod Trigriopus japonicus consumed by red sea bream larvae Pagrus major. Bull. Nagasaki Prefect. inst. fish., 2, 101-104.

Kitajima C., 1979. Aquisition of fertilized eggs and mass culture of juveniles of red sea bream Pagrus major. Special report of Nagasaki Prefect. Inst. Fish., 5, 92 p.

Kitajima C., Fukcsho K., Iwamoto H., Yаw'Awoto H., 1976. Amount of the rotifer Brachionus plicatilis consumed by red sea bream larvae Pagrus major. Bull. Nagasaki Prefect. Inst. Fish., 2, 105-112.

Keñg J., 1974. Travaux réalisés sur l'alimentation de la daurade dorée (Sparus auratus) par C.S.M.E. et S.A.R.B. Publications du C.N.E.X.O., Série Actes de Colloques, 1, 313-324.

Porter C., 1979. Cage culture of Sparus auratus in the Gulf of Aquaba, 1975-1979. Communication présentée lors de la 4" session du C.O.P.R.A.Q., Barcelone, 16-19 octobre 1979.

Rabe J.R., Echelle A.A., Schlichting E., 1973. Viability of algac in the digestive tracts of two cyprinodontids. Progress. fish. Cult., 35, 147-149.

Ramos J., 1978. Experiencias de cultivo de dorada Sparus auratus L. en Tanques Informes Tecnicos del instituto de investigaciones pesqueras, 55, $20 \mathrm{p}$.

RENE F., 1974 a. Données comparatives des phases de la croissance chez Sparus auratus en élevage intensif d'alevins obtenus par la pêche et le contrôle de la reproduction. Publications du C.N.E.X.O., série Actes de Colloques, 1, 305-312.

RENE F., 1974 b. Données sur la croissance de Sparus auratus Linné 1758 acquises par l'élevage en conditions intensives d'alevins obtenus par contrôle de la reproduction de 62 jours $(0,14 \mathrm{~g})$ à 263 jours $(100 \mathrm{~g})$. Publications du C.N.E.X.O., série Actes de Colloques, 1, 279-291.

Tesseyre C., Divanach P., Kentouri M., 1978. Procédé d'élevage de poisson, produit obtenu et dispositif. Brevet d'invention $78-34303$ A.N.V.A.R. France, 15 p. 